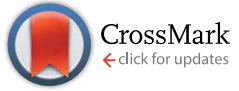

Cite this: RSC Adv., 2017, 7, 12704

Received 23rd January 2017

Accepted 17th February 2017

DOI: 10.1039/c7ra01003f

rsc.li/rsc-advances

\title{
Transport properties of a three-shell icosahedral matryoshka cluster: a first-principles study $\dagger$
}

\author{
Lu Zhang, ${ }^{a}$ Jing Huang, ${ }^{\text {*b }}$ Weiyi Wang, ${ }^{a}$ Qunxiang Li ${ }^{\star a}$ and Jinlong Yang ${ }^{a}$
}

Extensive efforts have been devoted to explore transport behaviors through various molecules and clusters, which are promising building blocks in molecular electronics. Here, we examine the spin-polarized electronic structures and transport properties of a three-shell icosahedral matryoshka cluster, $\mathrm{Pb} \propto \mathrm{Mn}_{12} \mathrm{aPb}_{20}$, by performing density functional theory calculations combining with non-equilibrium Green's function method. Theoretical results clearly reveal that, twelve $\mathrm{Mn}$ atoms in the middle layer anti-ferromagnetically couple with the center $\mathrm{Pb}$ atom and $\mathrm{Pb}$ atoms in the outlayer, while the $\mathrm{Pb} @ \mathrm{Mn}_{12} \mathrm{QPb}_{20}$ cluster still has a huge magnetic moment of 28.0 bohr magneton, mainly contributed by these $\mathrm{Mn}$ atoms. The calculated spin-resolved transmission spectra of the proposed $\mathrm{Pb}\left(\mathrm{Mn}_{12} \mathrm{aPb}_{20}\right.$ junctions exhibit robust spin filtering effect, which is not sensitive to the anchoring distance and the adopted electrode materials, and the conductance through the cluster under the small bias voltage is mainly determined by the spin-up electrons. These findings indicate that this kind of three-shell matryoshka cluster with huge magnetic moment holds potential applications in molecular spintronic devices.

\section{Introduction}

Since Aviram and Ratner first proposed the assumption of the molecular rectifier through a molecular junction in $1974,{ }^{1}$ it took almost 20 years of technological developments to realize the first experiment resembling transport through single molecules. Fortunately, the field of molecular electronics has made significant progress in the past decades. ${ }^{2-4}$ Recently, combining the contemporary exploitation of the electron and spin degrees of freedom at single-molecule level, molecular spintronics has attracted considerable attention since it holds promise for the next generation of electronic devices with enhanced functionality and improved performance, and now it finds various applications in nanodevices, most notably in the logical and memory units of computers. ${ }^{4-10}$

A variety of techniques, i.e. mechanical break junctions, electrochemical deposition, electromigration, electron beam lithography, shadow mask evaporation, scanning probe techniques, on-wire lithography and molecular rulers, have been developed to build molecular junctions, ${ }^{3,11}$ based on various

${ }^{a}$ Hefei National Laboratory for Physical Sciences at the Microscale, Synergetic Innovation Center of Quantum Information and Quantum Physics, University of Science and Technology of China, Hefei, Anhui 230026, China. E-mail: liqun@ustc. edu.cn

${ }^{b}$ School of Materials and Chemical Engineering, Anhui Jianzhu University, Hefei, Anhui 230601, China.E-mail: jhuang@ustc.edu.cn

$\dagger$ Electronic supplementary information (ESI) available: The structural models for the free cluster, the cluster adsorption on $\mathrm{Au}(100)$ surface at the bridge site, and the cluster is sandwiched between Au electrodes. See DOI: 10.1039/c7ra01003f promising candidates, such as organometallic wire, ${ }^{12-14}$ metal phthalocyanines, ${ }^{15,16}$ metal clusters, ${ }^{17-19}$ magnetic $\mathrm{C}_{28}$ molecule $^{20}$ and molecular magnets. ${ }^{21,22}$ Various spin devices including spin filtering, spin valves, and spin crossover, have been successfully demonstrated in experiments or have been theoretically predicted. ${ }^{23-25}$ Among various candidates, metal clusters have many special interesting controlled properties, which can be altered by the geometric structure, symmetry, cluster size, composition and the charged states. Previous investigations mainly focus on the physicochemical properties of metal clusters. ${ }^{26,27}$ For example, the magnetic moments of transition metal (TM)-doped $\mathrm{Au}_{6}$ cluster vary from 0 to $4.0 \mu_{\mathrm{B}}$ (Bohr magneton) by doping different TM atoms. The transport behavior of this kind of TM-doped clusters is limited so far. Our previous study has demonstrated that the spin-resolved trans-

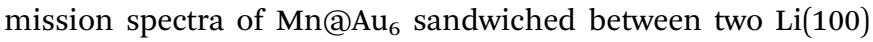
electrodes exhibit robust spin filtering effect. ${ }^{28}$ Clearly, more attention should be paid for their transport property.

Recently, several groups have proposed novel magnetic metal clusters, ${ }^{26,27,29,30}$ namely, three-shell icosahedral matryoshka clusters with the highest symmetry of $I_{\mathrm{h}}$ point group, which are promising building blocks in novel magnetic materials and devices since they have large magnetic moments, moderate HOMO-LUMO gaps, and weak inter-cluster interaction energies. ${ }^{29}$ Unfortunately, to our knowledge, their transport behaviors have not been visited so far. Previous reports mainly focused on the geometric structure, electronic and magnetic properties. Here, based on extensive density functional theory calculations combined with nonequilibrium Green's function 
formalism, we explore the spin-polarized transport properties through $\mathrm{Pb} @ \mathrm{Mn}_{12} @ \mathrm{~Pb}_{20}$ cluster. This three-shell icosahedral matryoshka cluster has a huge magnetic moment of $28.0 \mu_{\mathrm{B}}$ (Bohr magneton), mainly contributed by the $\mathrm{Mn}$ atoms, although the $\mathrm{Mn}$ atoms in the cluster anti-ferromagnetically couple with the $\mathrm{Pb}$ atoms in subunits. According to the calculated spin-resolved transmission spectra, we find that these $\mathrm{Pb} @ \mathrm{Mn}_{12} @ \mathrm{~Pb}_{20}$-based junctions exhibit robust spin filtering effect, and the conductance through the cluster is mainly determined by the spin-up electrons under the small bias voltage.

\section{Computational methods}

In our calculations, geometry optimizations, electronic structures and transport properties of $\mathrm{Pb} @ \mathrm{Mn}_{12} @ \mathrm{~Pb}_{20}$ cluster are calculated by using density functional theory calculations ${ }^{\mathbf{3 1}}$ combined with non-equilibrium Green's function method, which is implemented in ATK package. ${ }^{32,33}$ The interaction between ionic cores and valence electrons is modeled with Troullier-Martins nonlocal pseudopotential. The generalized gradient approximation (GGA) in the PW91 form is used to describe the exchange and correlation energy, ${ }^{34}$ since the trends and predictions from DFT are clear and understandable at the PW91 level. An energy cutoff is set to be 150 Ry for the real-space grid on which the Poisson equation is solved. This is sufficient for electronic structure and transport calculations of $\mathrm{Pb} @ \mathrm{Mn}_{12} @ \mathrm{~Pb}_{20}$ cluster (see ESI $\dagger$ ). Here, double-zeta plus polarization basis is used for all $\mathrm{Pb}$ and $\mathrm{Mn}$ atom in clusters, while single-zeta plus polarization basis is used for electrodes (i.e. $\mathrm{Au}$ and $\mathrm{Cu}$ ) to save the computational cost.

The spin-polarized transmission coefficients of these proposed molecular junctions are calculated by

$$
T_{\sigma}(E, V)=\operatorname{Tr}\left[\Gamma_{\mathrm{L}} G_{\sigma} \Gamma_{\mathrm{R}} G_{\sigma}^{+}\right]
$$

Here, $\sigma$ stands for the spin-up $(\uparrow)$ and spin-down $(\downarrow)$ channels. $G_{\sigma}$ is the spin-dependent Green's function of the scattering region, $\Gamma_{\mathrm{L} / \mathrm{R}}$ is the coupling matrix between the scattering region and the left/right electrode. The current-voltage $(I-V)$ curves are calculated by the Landauer-Buttiker formula,

$$
I(V)=\frac{e}{h} \int T_{\sigma}(E, V)\left[f\left(E-\mu_{\mathrm{L}}\right)-f\left(E-\mu_{\mathrm{R}}\right)\right] \mathrm{d} E,
$$

in which, the $f\left(E-\mu_{\mathrm{L}(\mathrm{R})}\right)$ is Fermi-Dirac function, while $\mu_{\mathrm{L}(\mathrm{R})}$ stands for the chemical potential of left/right electrode.

\section{Results and discussion}

\subsection{Free Pb@Mn $12 @ \mathrm{~Pb}_{20}$ cluster}

We start with performing spin-polarized DFT calculations for the geometric, magnetic and electronic properties of the free $\mathrm{Pb} @ \mathrm{Mn}_{12} @ \mathrm{~Pb}_{20}$ cluster as benchmark. Fig. 1(a) shows the optimized structure. The most energy stable configuration of $\mathrm{Pb} @ \mathrm{Mn}_{12} @ \mathrm{~Pb}_{20}$ cluster displays a perfect icosahedral symmetrical three-shell matryoshka doll structure. It is an outer $\mathrm{Pb}_{20}$ regular dodecahedron encapsulating a $\mathrm{Mn}_{12}$ icosahedron,

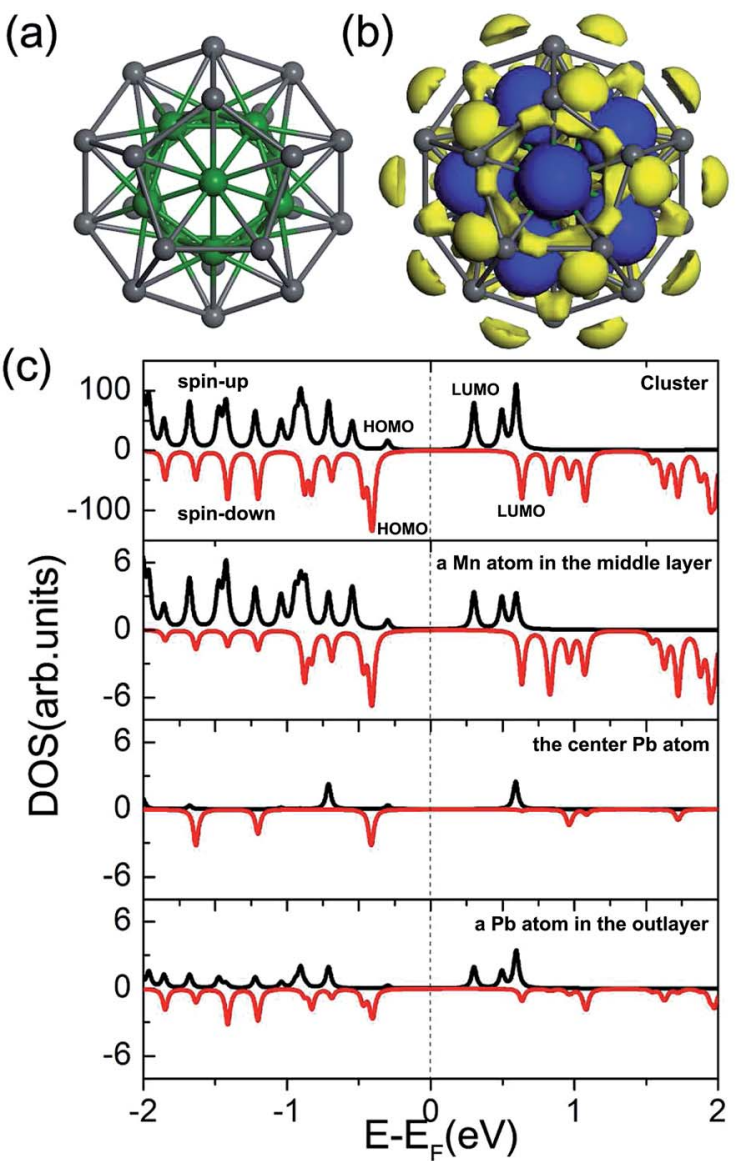

Fig. 1 (a) The optimized structure of $\mathrm{Pb} a \mathrm{Mn}_{12}\left(\mathrm{aPb}_{20}\right.$ cluster, (b) its spin density distribution. (c) DOS of the $\mathrm{Pb} @ \mathrm{Mn}_{12} @ \mathrm{aPb}_{20}$ cluster.

which in turn encapsulates a center $\mathrm{Pb}$ atom. Note that this cluster has icosahedral symmetry ( $I_{\mathrm{h}}$ group), but its shape is not an icosahedron but a pentadodecahedron. The optimized $\mathrm{Pb}-$ $\mathrm{Pb}$ and $\mathrm{Mn}-\mathrm{Mn}$ distances are about 3.29 and $2.75 \AA$, respectively. To determine the ground state of $\mathrm{Pb} @ \mathrm{Mn}_{12} @ \mathrm{~Pb}_{20}$ cluster, different magnetic configurations are examined, and we find that the calculated magnetic moments are not sensitive to the adopted functionals. The $\mathrm{Pb} @ \mathrm{Mn}_{12} @ \mathrm{~Pb}_{20}$ cluster prefers to the spin-polarized state, and the total magnetic moment (MM) is predicted to be $28.0 \mu_{\mathrm{B}}$, while the atomic $\mathrm{MM}$ of $\mathrm{Mn}$ and $\mathrm{Pb}$ in the midderlayer and outlayer is about 3.49 and $-0.64 \mu_{\mathrm{B}}$, while the center $\mathrm{Pb}$ atom has $-0.99 \mu_{\mathrm{B}}$, respectively. Clearly, that the total MM is mainly contributed by the twelve Mn atoms, which anti-ferromagnetically couple with the center $\mathrm{Pb}$ atom and $\mathrm{Pb}$ atom in the outlay. Fig. 1(b) presents the spin density of $\mathrm{Pb} @ \mathrm{Mn}_{12} @ \mathrm{~Pb}_{20}$ cluster, here, the blue and yellow regions stand for the spin-up and spin-down densities, respectively. It is clear that the spin density mainly localizes around Mn atoms, which verifies that $\mathrm{Mn}$ atoms give the dominative contribution to the predicted $\mathrm{MM}$, while there is distinguishable spin density around these $\mathrm{Pb}$ atoms. These obtained geometric parameters and MMs agree well with the foregoing reports, ${ }^{29}$ indicating that the computational methods and parameters adopted here are suitable to describe $\mathrm{Pb} @ \mathrm{Mn}_{12} @ \mathrm{~Pb}_{20}$ cluster. 
To clearly illustrate the origin of the predicted MMs, we calculate the total density of states (DOS) of $\mathrm{Pb} @ \mathrm{Mn}_{12} @ \mathrm{~Pb}_{20}$ cluster, the partial DOS of one Mn atom in the middle layer, the center $\mathrm{Pb}$ atom and one $\mathrm{Pb}$ in the outlayer, and plot them in Fig. 1(c). Clearly, all calculated DOS are obviously spinpolarized. The spin-up states are filled more than the spindown states for $\mathrm{Pb} @ \mathrm{Mn}_{12} @ \mathrm{~Pb}_{20}$ cluster and for the $\mathrm{Mn}$ atom, while this observation reverses for two $\mathrm{Pb}$ atoms, which are consistent with the predicted MMs. Note that, the positions of the highest occupied molecular orbital (HOMO) and the lowest unoccupied molecular orbital (LUMO) of $\mathrm{Pb} @ \mathrm{Mn}_{12} @ \mathrm{~Pb}_{20}$ cluster for the spin-up and spin-down electrons are remarkably different. The HOMO (LUMO) of the spin-up electrons locates at about $-0.35(0.3)$, while they locate at $-0.4(0.65)$ for the spindown electrons. Then, the gaps between the HOMO and LUMO for two spin channels are also different, which are predicted to be 0.65 and $1.05 \mathrm{eV}$, respectively, although they are always underestimated at the GGA level. This predicted large MM and the remarkable difference in the electronic structures of the spin-up and spin-down states indicate that $\mathrm{Pb} @ \mathrm{Mn}_{12} @ \mathrm{~Pb}_{20}$ cluster is a possible candidate for designing molecular devices.

\section{$3.2 \mathrm{~Pb} @ \mathrm{Mn}_{12} @ \mathrm{~Pb}_{20}$ cluster on substrate}

Before exploring the spin-polarized transport, we firstly examine the substrate effect on the geometric and magnetic properties of $\mathrm{Pb} @ \mathrm{Mn}_{12} @ \mathrm{~Pb}_{20}$ cluster. Here, $\mathrm{Au}(100)$ surface is taken as an example, which is simulated by a $(6 \times 6)$ supercell cell with periodic boundary conditions. The lattice constant in $\mathrm{Au}(100)$ slab model is $4.08 \AA$, taken from experimental distance. The cluster adsorbing on the hollow, bridge, and atop sites are considered. We find that the molecule prefers to adsorb $\mathrm{Au}(100)$ surface at the bridge site via a five-membered $\mathrm{Pb}$ ring, as shown in Fig. 2(a). The averaged vertical distance between $\mathrm{Au}(100)$ surface and the bottom five-member ring of $\mathrm{Pb} @ \mathrm{Mn}_{12} @ \mathrm{~Pb}_{20}$ cluster is about $2.65 \AA$, while the average $\mathrm{Pb}-\mathrm{Au}$ bond length is $3.00 \AA$ A. This relatively small separation implies that the cluster$\mathrm{Au}$ interaction is rather strong, which results in charge transfer between the cluster and Au surface. Fig. 2(b) plots the electron density difference, in which the blue and yellow regions represent charge accumulation and depletion, respectively. It is clear that the charge mainly transfers from the bottom part of $\mathrm{Pb} @ \mathrm{Mn}_{12} @ \mathrm{~Pb}_{20}$ cluster to these $\mathrm{Au}$ atoms locating around the anchoring interface. This observation is verified by the Mulliken charge analysis, and the net charge on the adsorbed $\mathrm{Pb} @ \mathrm{Mn}_{12} @ \mathrm{~Pb}_{20}$ cluster on $\mathrm{Au}(100)$ surface is predicted to be about $+1.41|e|$ (here $e$ is the electron charge).

The $\mathrm{MM}$ of the $\mathrm{Pb} @ \mathrm{Mn}_{12} @ \mathrm{~Pb}_{20}$ cluster on $\mathrm{Au}(100)$ surface is predicted to be $30.3 \mu_{\mathrm{B}}$, while the atomic $\mathrm{MM}$ of the labeled Mn1, Mn2, and Pb atoms in Fig. 2(a) is about 3.0, 2.8, and -0.2 $\mu_{\mathrm{B}}$, respectively. These results mean that the cluster magnetism is slightly enhanced due to the presence of $\mathrm{Au}(100)$ surface. This observation is totally distinct from a CoPc molecule adsorption on $\mathrm{Au}(111)$ surface, in which the molecular magnetism is quenched due to the molecule-substrate interaction. ${ }^{35}$ To understand this result, Fig. 2(c) shows the calculated spin density. Clearly, the spin density still mainly localizes around (a)
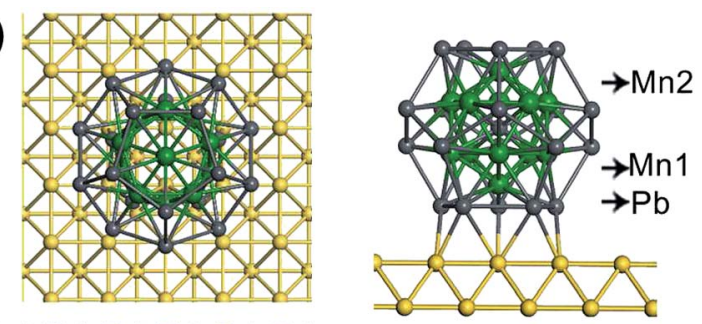

(b)

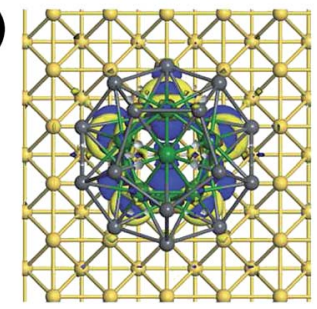

(c)
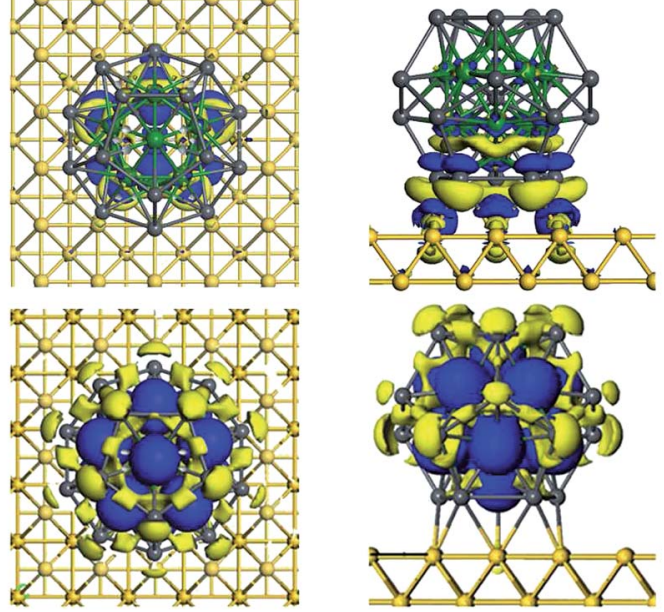

Fig. 2 (a) Adsorption structure of $\mathrm{Pb} @ \mathrm{Mn}_{12} \mathrm{aPb}_{20}$ cluster on $\mathrm{Au}(100)$ surface. (b) Electron density difference, here, the blue and yellow colors stand for the charge accumulation and depletion, respectively. (c) Spin density distribution, here, the blue and yellow colors label for the spin-up and spin-down electrons, respectively.

twelve Mn atoms, which still anti-ferromagnetically couple with these $\mathrm{Pb}$ atoms. The main reason for the enhanced cluster magnetism is that from these $\mathrm{Pb}$ atomic $\mathrm{MMs}$ at the bottom five-member are almost quenched due to the observed interfacial charge transfer, as shown in Fig. 2(b).

Fig. 3 plots the calculated total DOS of the $\mathrm{Pb} @ \mathrm{Mn}_{12} @ \mathrm{~Pb}_{20}$ cluster on $\mathrm{Au}(100)$ surface and the corresponding partial DOS of the $\mathrm{Pb}, \mathrm{Mn} 1$ and $\mathrm{Mn} 2$ atoms, which are labeled in Fig. 2(a). Compared with the free $\mathrm{Pb} @ \mathrm{Mn}_{12} @ \mathrm{~Pb}_{20}$ cluster's results, the total DOS peaks are obviously broadened due to the rather strong cluster-substrate interaction. The partial DOS of the $\mathrm{Pb}$ atom in the bottom five-membered ring is virtually spinrestricted, which leads to the $\mathrm{Pb}$ atomic $\mathrm{MM}$ quenching.

\subsection{Spin-polarized transport properties}

Now we turn to examine the spin-polarized transport properties of $\mathrm{Pb} @ \mathrm{Mn}_{12} @ \mathrm{~Pb}_{20}$ cluster. In a typical scanning tunneling microscopy experiment for single molecule transport measurements, a molecule always adsorbs on a flat surface (as one electrode), then a tip (act as the other electrode) is used to measure the current through the molecule. To mimic this situation, the $\mathrm{Pb} @ \mathrm{Mn}_{12} @ \mathrm{~Pb}_{20}$ cluster is sandwiched between two $\mathrm{Au}(100)$ electrodes. Fig. 4(a) illustrates the proposed junction, in which the left and right $\mathrm{Au}$ electrodes are modeled with two $(5 \times 5)$ supercells. This two-probe system can be divided into three parts: the left and right electrodes, and the central 


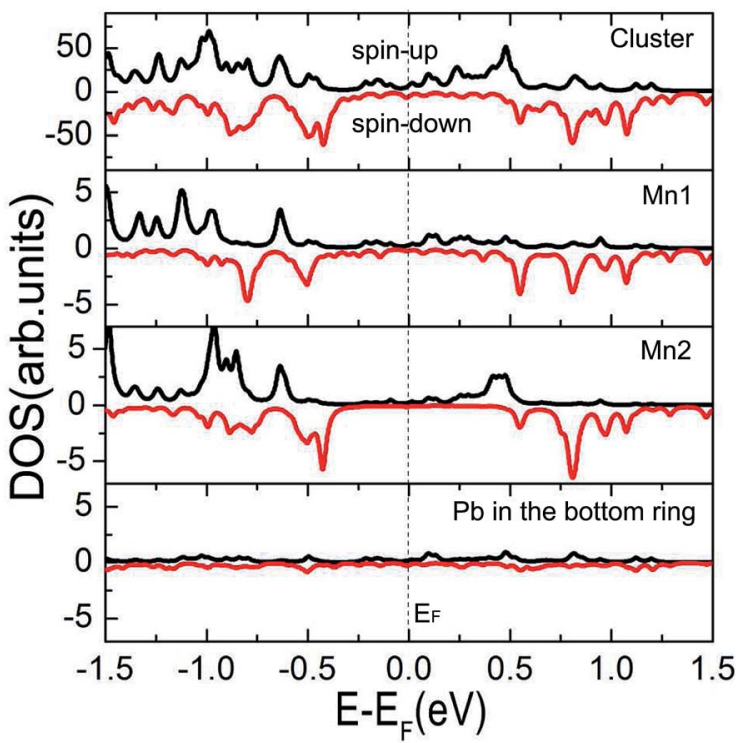

Fig. 3 Total DOS of the $\mathrm{Pb}\left(\mathrm{Mn}_{12} \mathrm{aPb}_{20}\right.$ cluster on $\mathrm{Au}(100)$ surface and the partial DOS of one $\mathrm{Pb}, \mathrm{Mn1}$, and $\mathrm{Mn} 2$ atoms, which are labeled in Fig. 2(a).

(a)
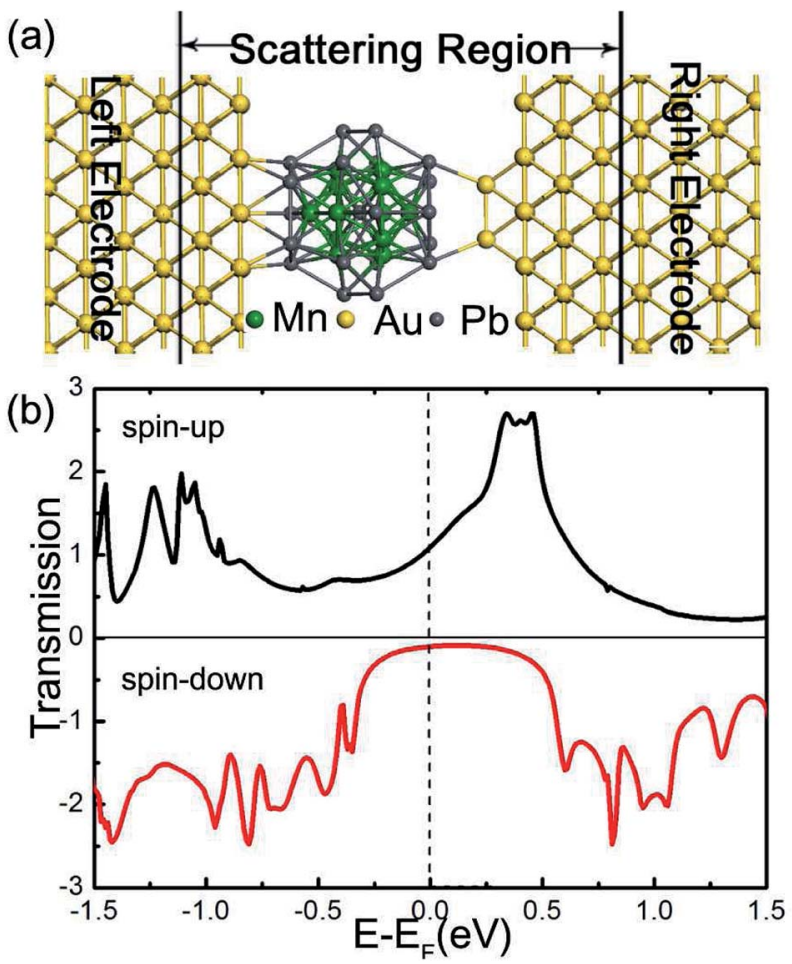

Fig. 4 (a) Proposed molecular junction, in which the Pb@Mn $\mathrm{Mn}_{12} \mathrm{aPb}_{20}$ cluster is sandwiched between two Au(100) electrodes. (b) Zero-bias energy dependent spin-resolved transmission spectra. Here, the black and red curves stand for the transmission curves for the spin-up and spin-down electron, respectively.

scattering region which includes $\mathrm{Pb} @ \mathrm{Mn}_{12} @ \mathrm{~Pb}_{20}$ cluster, two surface layers of the left electrode, and two Au adatoms (acting as a tip) on three surface layers of the right electrode. $\mathrm{The} \mathrm{Au}-\mathrm{Pb}$ anchoring distance is optimized to be about $2.65 \mathrm{~A}$, which indicates that the cluster is strongly coupled with electrode surfaces. The spin-resolved transmission spectra versus $E-E_{\mathrm{F}}$ under zero-bias voltage of the junction is illustrated in Fig. 4(b). Here, the vertical black dotted line represents the position of the Fermi level, which is already set to zero for clarity, while the black and red solid lines stand for the transmission curves of the spin-up and spin-down electrons, respectively. It is clear that the conductance behavior of two spin channels is remarkably different. For instance, there is a broad and significant transmission peak locating around $0.4 \mathrm{~V}$ for the spin-up electrons, while for the spin-down electrons, the transmission coefficients within the energy range from -0.2 to $0.3 \mathrm{eV}$ are small. The conductance of the spin-up electrons at the Fermi level is predicted to be $1.33 G_{0}$ ( $G_{0}$ denotes the quantum constant, and equals to $e^{2} / h$ ), which is significantly larger than that of the spindown electrons $\left(0.16 G_{0}\right)$. That to say, the spin-resolved transmission spectra of $\mathrm{Pb} @ \mathrm{Mn}_{12} @ \mathrm{~Pb}_{20}$ molecular junction exhibit robust transport spin polarization characteristics. To quantify this remarkable difference for the spin-up and spin-down electrons, we defined spin filter efficiency (SFE) as: $\mathrm{SFE}=\left(T_{\uparrow}-T_{\downarrow}\right) /$ $T_{\uparrow}$. Here, $T_{\uparrow}\left(E_{\mathrm{F}}\right)$ and $T_{\downarrow}\left(E_{\mathrm{F}}\right)$ stand for the transmission coefficients of spin-up and spin-down electrons at the Fermi level, respectively. The SFE is predicted to be about $88.0 \%$. Clearly, the conductance behavior through $\mathrm{Pb} @ \mathrm{Mn}_{12} @ \mathrm{~Pb}_{20}$ molecular junction is mainly governed by the spin-up electrons under the small bias voltage. This observation is similar to the results of europium-cyclooctatetraene, ${ }^{36} \mathrm{FeN}_{4}$ complexes, ${ }^{37-39}$

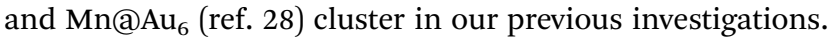

This observed spin filtering effect can be easily understood by exploring the local density of states (LDOS) of the $\mathrm{Pb} @ \mathrm{Mn}_{12} @ \mathrm{~Pb}_{20}$ molecular junction around the Fermi level. The spatial distribution of the LDOS at the Fermi level is plotted in Fig. 5(a), and the calculated DOS of the scattering region is presented in Fig. 5(b), respectively. It is clear that the LDOS of the spin-up electrons delocalized over around the whole

(a)
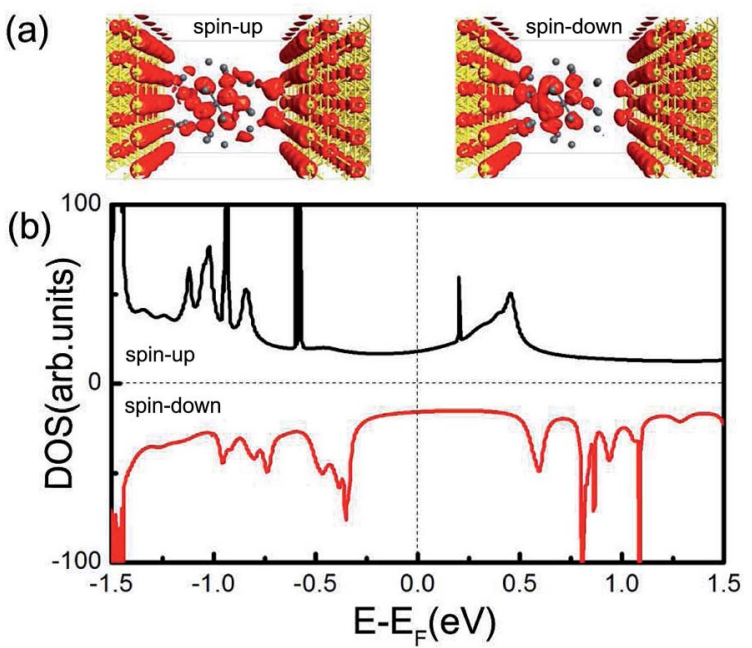

Fig. 5 (a) Spatial profile of the LDOS at the Fermi level for spin-up and spin-down electrons. (b) Spin-resolved DOS of the Pb@Mn $\mathrm{n}_{12} \mathrm{CPb}_{20}$ cluster in the proposed junction for spin-up and spin-down electrons. 
molecule junction, which provides a good transport channel. On the contrary, as for the spin-down electrons, the LDOS mainly localize at the right region of the cluster, and resulting in a small conductance at the Fermi level. Actually, the positions of these DOS peaks correspond well with the transmission peaks. The LUMO of the spin-up channel is localized, which does not contribute an obvious transmission peak. The transmission peak at $0.4 \mathrm{~V}$ is contributed by the perturbed LUMO+1 and $\mathrm{LUMO}+2$.

The spin-polarized current-voltage $(I-V)$ curves through $\mathrm{Pb} @ \mathrm{Mn}_{12} @ \mathrm{~Pb}_{20}$ molecular junction are calculated and plotted in Fig. 4. In our calculations, at each bias voltage, the current is determined self-consistently Landauer-Buttiker formula under the non-equilibrium condition, black and red lines stand for spin-up and spin-down electrons, respectively. Clearly, the current of the spin-up electrons is significant larger than that of the spin-down ones. To quantify the current difference between the spin-up and spin-down electrons, the ratio of currents is defined as $R(V)=\left|I_{\uparrow} / I_{\downarrow}\right|$. The predicted $R$ is up to 14 within the examined bias voltage range, which is readily measurable in experiments. In other words, the $I-V$ results through $\mathrm{Pb} @ \mathrm{Mn}_{12} @ \mathrm{~Pb}_{20}$ molecular junction confirm this proposed spin-filtering device can works at finite bias voltage (Fig. 6).

In general, the distance between the molecule and the electrode is variable in experiments. ${ }^{40} \mathrm{We}$ examine the dependence of transport properties of the $\mathrm{Pb} @ \mathrm{Mn}_{12} @ \mathrm{~Pb}_{20}$ junction on the different $\mathrm{Au}-\mathrm{Pb}$ anchoring distances. Even the end $\mathrm{Au}-\mathrm{Pb}$ distance is elongated to be $3.0 \AA$, the main features of the spinresolved transmission curves through the junction do not significantly change. The $T_{\uparrow}$ and $T_{\downarrow}$ are predicted to be 1.08 and $0.10 G_{0}$, and the corresponding SFE is about $90.7 \%$. That is to say, the transport spin polarization characteristics is not sensitive to the end $\mathrm{Au}-\mathrm{Pb}$ anchoring distance.

Note that the exact anchoring configuration of molecular devices in experiments is a 'blackbox' so far. ${ }^{41-45}$ Here, we propose two $\mathrm{Pb} @ \mathrm{Mn}_{12} @ \mathrm{~Pb}_{20}$-based junctions with different anchoring configurations between $\mathrm{Li}$ and $\mathrm{Cu}(100)$ electrodes to examine whether the spin filtering effect exists or not. Three Li

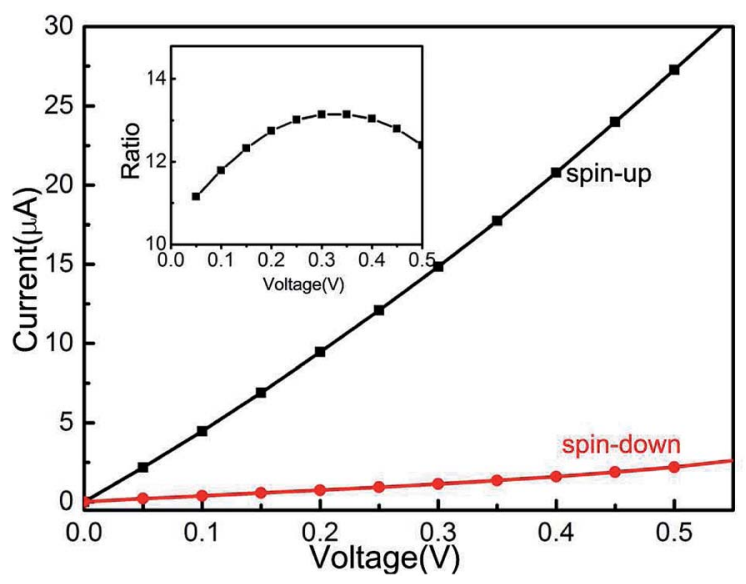

Fig. 6 Calculated spin-resolved I-V curves of the Pb@Mn $\mathrm{Mn}_{12} \mathrm{aPb}_{20}$ molecular junction. Here, the inset denotes the rectification ratio versus bias voltage.

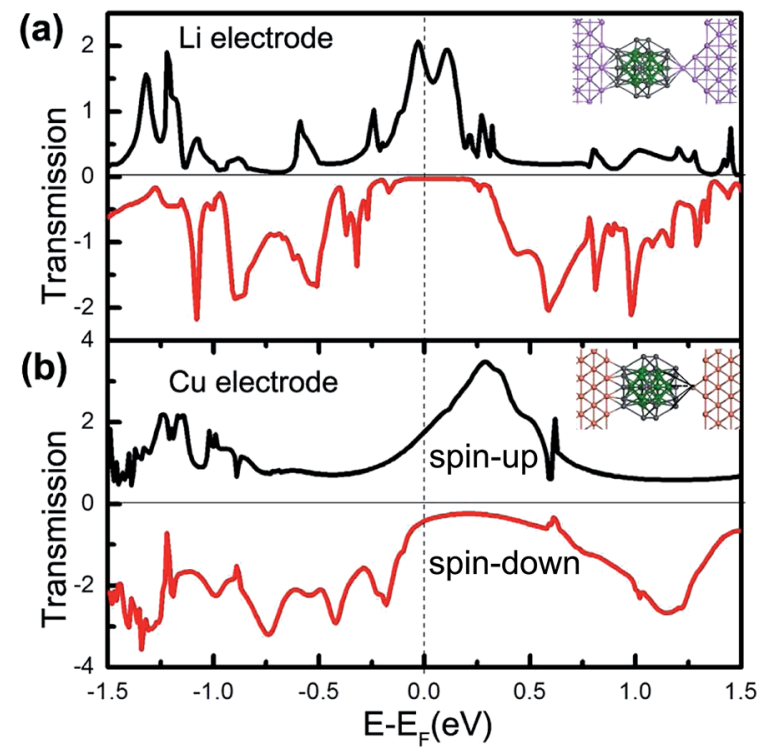

Fig. 7 Zero-bias energy dependent spin-resolved transmission spectra of the $\mathrm{Pb}\left(\mathrm{Mn}_{12} \mathrm{aPb}_{20}\right.$ cluster connected to different electrodes. (a) Li(100) electrode, (b) $\mathrm{Cu}(100)$ electrode. Here, insets stand for two examined molecular junctions.

atoms and on $\mathrm{Cu}$ atom are pulled outwards the surfaces for the $\mathrm{Li}$ and $\mathrm{Cu}$ electrodes to simulate the different anchoring configurations, as schematically illustrated with inserts in Fig. 7(a) and (b), and the corresponding zero-bias energy dependent spin-resolved transmission spectra of the $\mathrm{Pb} @ \mathrm{Mn}_{12} @ \mathrm{~Pb}_{20}$ cluster connected to $\mathrm{Li}$ and $\mathrm{Cu}$ electrodes are plotted, respectively. It is clear that the transport spin polarization characteristics are still robust. For example, around the Fermi level, there is a significant and broad transmission peak for the spin-up electrons, and the $T_{\uparrow}$ is $1.75 G_{0}$ for the Li electrodes, while for the spin-down electrons, their transmission coefficients are small, and the corresponding $T_{\downarrow}$ is about $0.03 G_{0}$. The SFE for junction with $\mathrm{Li}$ and $\mathrm{Cu}$ electrodes is predicted to be about $98.3 \%$ and $76.1 \%$, respectively. As for the same electrodes, such as $\mathrm{Au}$ and $\mathrm{Li}$ as examples, we also observe the obvious transport spin polarization feature in the zero-bias energy dependent spin-resolved transmission spectra (see ESI $\dagger$ ). That is to say, the predicted spin-filtering effect is not sensitive to the detailed anchoring configurations and the adopted electrode materials. This observation highlights this kind of magnetic three-shell icosahedral matryoshka cluster with huge MM for promising applications in molecular spintronic devices.

\section{Conclusions}

Based on DFT calculations combined with the NEGF technique, the electronic structures, magnetic and spin transport properties of the $\mathrm{Pb} @ \mathrm{Mn}_{12} @ \mathrm{~Pb}_{20}$ cluster are explored. Theoretical results indicate that the $\mathrm{Pb} @ \mathrm{Mn}_{12} @ \mathrm{~Pb}_{20}$ cluster has a huge magnetic moment of $28.0 \mu_{\mathrm{B}}$, mainly contributed twelve $\mathrm{Mn}$ atoms in the middle layer, which anti-ferromagnetically couple 
to the central $\mathrm{Pb}$ atom and the $\mathrm{Pb}$ atoms in the outlayer. The zero-bias transmission coefficients of the spin-up electrons through $\mathrm{Pb} @ \mathrm{Mn}_{12} @ \mathrm{~Pb}_{20}$ cluster sandwiched between two $\mathrm{Au}(100)$ electrodes are significantly larger than that of the spindown electrons. The spin filter efficiency is predicted to be $83.2 \%$. Moreover, the proposed model device can stably work at finite bias voltage, and this observed spin filtering effect is not sensitive to the anchoring distance and the adopted electrode materials. These findings indicate that this kind of magnetic three-shell icosahedral matryoshka cluster with huge MM can be used to design spin-filtering device.

\section{Acknowledgements}

This work was partially supported by the National Key Basic Research Program (No. 2014CB921101), by the State Key RD Fund of China (No. 2016YFA0200604), by the National Natural Science Foundation of China (No. 21473168 and 11634011), by the Innovative Program of Development Foundation of Hefei Center for Physical Science and Technology (Q. L.). J. H. thanks the Natural Science Foundation of the Anhui Higher Education Institutions (No. KJ2016A144) and the Natural Science Foundation of Anhui Province (No. 1408085QB26). Computational resources have been provided by CAS, Shanghai and USTC Supercomputer Centers.

\section{References}

1 A. Aviram and M. A. Ratner, Chem. Phys. Lett., 1974, 29, 277. 2 M. Kiguchi and S. Kaneko, ChemPhysChem, 2012, 13, 1116.

3 C. C. Jia, A. Migliore, N. Xin, S. Y. Huang, J. Y. Wang, Q. Yang, S. P. Wang, H. L. Chen, D. M. Wang, B. Y. Feng, Z. R. Liu, G. Y. Zhang, D. H. Qu, H. Tian, M. A. Ratner, H. Q. Xu, A. Nitzan and X. F. Guo, Science, 2016, 352, 1443-1445.

4 C. D. Frisbie, Science, 2016, 352, 1394-1395.

5 C. Joachim, J. K. Gimzewski and A. Aviram, Nature, 2000, 408, 541.

6 J. Chen, M. A. Reed, A. M. Rawlett and J. M. Tour, Science, 1999, 286, 1550.

7 M. Galperin, M. A. Ratner and A. Nitzan, Nano Lett., 2005, 5, 125.

8 S. Yasuda, T. Nakamura, M. Matsumoto and H. Shigekawa, J. Am. Chem. Soc., 2003, 125, 16430.

9 S. Yeganeh, M. Galperin and M. A. Ratner, J. Am. Chem. Soc., 2007, 129, 13313.

10 E. Ruiz, Phys. Chem. Chem. Phys., 2014, 16, 14-22.

11 H. Ren, G. P. Zhang, N. Lin, L. Deng, Y. Luo and F. Huang, Phys. Chem. Chem. Phys., 2016, 18, 26586-26594.

12 L. Zhu, F. Zou, J. H. Gao, Y. S. Fu, G. Y. Gao, H. H. Fu, M. H. Wu, J. T. Lü and K. L. Yao, Nanotechnology, 2015, 26, 315201.

13 A. Nitzan and M. A. Ratner, Science, 2003, 300, 1384-1389.

14 C. C. Kaun and T. Seideman, Phys. Rev. B: Condens. Matter Mater. Phys., 2008, 77, 033414.

15 I. Aviv-Harel and Z. Gross, Coord. Chem. Rev., 2011, 255, 717736.
$16 \mathrm{H}$. Palmer, in Molecular electronic structures of transition metal complexes I, Springer, 2011, pp. 49-89.

17 X. X. Zhang, Y. Wang, H. P. Wang, L. Alane, G. Gantefoer, K. H. Bowen, J. U. Reveles and S. N. Khanna, J. Am. Chem. Soc., 2013, 135, 4856-4861.

18 K. Damianos, P. Solokha and R. Ferrando, RSC Adv., 2013, 3, 9419-9430.

19 S. Stegmaier and T. F. Fässler, Angew. Chem., Int. Ed., 2012, 51, 2647-2650.

20 K. Xu, J. Huang, Z. Y. Guan, Q. X. Li and J. L. Yang, Chem. Phys. Lett., 2012, 535, 111-115.

21 F. K. Sheong, W. J. Chen, H. Kim and Z. Y. Lin, Dalton Trans., 2015, 44, 7251-7257.

22 J. Huang, R. Xie, W. Y. Wang, Q. X. Li and J. L. Yang, Nanoscale, 2016, 8, 609-616.

23 L. H. Yu and D. Natelson, Nano Lett., 2004, 4, 79.

24 A. Saffarzadeh, J. Appl. Phys., 2008, 103, 083705.

25 T. Xu, J. Huang and Q. X. Li, Chin. J. Chem. Phys., 2014, 27, 668-673.

26 J. L. Wang, J. Bai, J. Jellinek and X. C. Zeng, J. Am. Chem. Soc., 2007, 129, 4110.

27 S. Stegmaier and T. F. Fässler, J. Am. Chem. Soc., 2011, 133, 19758.

28 J. Huang, W. Y. Wang, S. F. Yang, Q. X. Li and J. L. Yang, Chem. Phys. Lett., 2013, 590, 111-115.

29 X. M. Huang, J. J. Zhao, Y. Su, Z. F. Chen and R. B. King, Sci. Rep., 2014, 4, 6915.

30 M. J. Moses, J. C. Fettinger and B. W. Eichhorn, Science, 2003, 300, 778.

31 J. M. Soler, E. Artacho, J. D. Gale, A. García, J. Junquera, P. Ordejón and D. Sánchez-Portal, J. Phys.: Condens. Matter, 2002, 14, 2745-2779.

32 J. Taylor, H. Guo and J. Wang, Phys. Rev. B: Condens. Matter Mater. Phys., 2001, 63, 245407.

33 M. Brandbyge, J.-L. Mozos, P. Ordejón, J. Taylor and K. Stokbro, Phys. Rev. B: Condens. Matter Mater. Phys., 2002, 65, 165401.

34 J. P. Perdew and Y. Wang, Phys. Rev. B: Condens. Matter Mater. Phys., 1992, 45, 13244.

35 A. D. Zhao, Q. X. Li, L. Chen, H. J. Xiang, W. H. Wang, S. Pan, B. Wang, X. D. Xiao, J. L. Yang, J. G. Hou and Q. S. Zhu, Science, 2005, 309, 1542.

36 K. Xu, J. Huang, S. L. Lei, H. B. Su, F. Y. C. Boey, Q. X. Li and J. L. Yang, J. Chem. Phys., 2009, 131, 104704.

37 J. Huang, W. Y. Wang, S. F. Yang, Q. X. Li and J. L. Yang, Nanotechnology, 2012, 23, 255202.

38 T. Miyamachi, M. Gruber, V. Davesne, M. Bowen, S. Boukari, L. Joly, F. Scheurer, G. Rogez, T. K. Yamada, P. Ohresser, E. Beaurepaire and W. Wulfhekel, Nat. Commun., 2012, 3, 938.

39 J. Huang, W. Y. Wang, S. F. Yang, H. B. Su, Q. X. Li and J. L. Yang, Chem. Phys. Lett., 2012, 539, 102-106.

40 B. Q. Xu and N. J. Tao, Science, 2003, 301, 1221.

41 A. N. Pasupathy, R. C. Bialczak, J. Martinek, J. E. Grose, L. A. K. Donev, P. McEuen and D. Ralph, Science, 2004, 306, 86-89.

42 T. Ono and K. Hirose, Phys. Rev. Lett., 2007, 98, 026804. 
43 A. N. Pasupathy, J. Park, C. Chang, A. V. Soldatov, S. Lebedkin, R. C. Bialczak, J. E. Grose, L. Donev, J. P. Sethna, D. C. Ralph and P. L. McEuen, Nano Lett., 2005, 5, 203-207.
44 Y. Yasutake, Z. J. Shi, T. Okazaki, H. Shinohara and Y. Majima, Nano Lett., 2005, 5, 1057-1060.

45 C. M. Guédon, H. Valkenier, T. Markussen, K. S. Thygesen, J. C. Hummelen and S. J. van der Molen, Nat. Nanotechnol., 2012, 7, 305-309. 